\title{
New insight on the quark condensate beyond the chiral limit
}

\author{
Ling-feng Chen, ${ }^{1, *}$ Zhan Bai, ${ }^{1, \dagger}$ Fei Gao ${ }^{2, \hbar}$ and Yu-xin Liu ${ }^{1,3,4, \S}$ \\ ${ }^{1}$ Department of Physics and State Key Laboratory of Nuclear Physics and Technology, Peking University, \\ Beijing 100871, China \\ ${ }^{2}$ Institut für Theoretische Physik, Universität Heidelberg, Philosophenweg 16, 69120 Heidelberg, Germany \\ ${ }^{3}$ Collaborative Innovation Center of Quantum Matter, Beijing 100871, China \\ ${ }^{4}$ Center for High Energy Physics, Peking University, Beijing 100871, China
}

(Received 9 June 2021; accepted 19 October 2021; published 29 November 2021)

\begin{abstract}
By solving the Dyson-Schwinger equations and analyzing the obtained mass function, we compute the quark condensate beyond the chiral limit with a cutoff independent definition. The definition explicitly separates the quadratic and logarithmic divergence from the condensate, even for very large quark mass. With this well-defined condensate, we then analyze the evolution of the condensate and its susceptibility with the current quark mass. The susceptibility shows a critical mass at about $\Lambda_{\mathrm{QCD}}$, which defines a transition boundary for internal hadron dynamics.
\end{abstract}

DOI: 10.1103/PhysRevD.104.094041

\section{INTRODUCTION}

Chiral symmetry and its breaking plays significant roles in QCD phase structure as well as hadron dynamics. The current mass of the quark inside a proton is only about $3-5 \mathrm{MeV}$, but the corresponding constituent quark acquires a mass of about $300-500 \mathrm{MeV}$ through interaction. This effect is called dynamical chiral symmetry breaking (DCSB) and is essential in the study of QCD properties (for a recent review, see Ref. [1]. See also Refs. [2-11]). In order to describe the transition from a dynamical chiral symmetric (DCS) phase to a DCSB phase, the chiral condensate, i.e., the expectation value of the composite operator $\bar{q} q$, is usually applied as the order parameter (see, e.g., Refs. [12-22]), and the chiral condensate is also related to many important problems such as the pion-nucleon sigma term [23], the cosmological constant [24], and thermodynamic quantities [25].

The chiral condensate and DCSB have been extensively studied in lattice QCD (see, e.g., Refs. [26-29]), functional renormalization group methods [19,30-35], DysonSchwinger equations (DSEs) [36-49], and effective field models such as the (Polyakov improved) NambuJona-Lasinio model [10,50-53] and quark meson model $[32,33,54,55]$. Among these theoretical approaches, the

\footnotetext{
*1fchen1130@pku.edu.cn

†baizhan@pku.edu.cn

${ }^{*}$ f.gao@thphys.uni-heidelberg.de

yxliu@pku.edu.cn
}

Published by the American Physical Society under the terms of the Creative Commons Attribution 4.0 International license. Further distribution of this work must maintain attribution to the author(s) and the published article's title, journal citation, and DOI. Funded by SCOAP.
DSE approach is a first-principle, nonperturbative continuum method. It is able to deal with the DCSB and confinement simultaneously and has been widely applied to study the hadron properties and the QCD phase transitions.

However, despite the great success of the theoretical studies, there is still ambiguity in the definition of the condensate, especially when the quark has a nonzero current mass, i.e., the explicitly chiral symmetry breaking (ECSB) is considered. The ECSB comes from the Higgs mechanism, which is significantly different from the DCSB mechanism in QCD. The effect of ECSB and its interference on DCSB is important for understanding the different mass generation mechanisms and their relations on the properties of quarks and hadrons (see, e.g., Refs. [11,41,56-58]). For instance, the pure DCSB effect with no current quark mass gives a massless pion, and after introducing the ECSB effect, more than 95\% of the pion mass comes from the interference. Managing the DCSB effect properly may also be quite significant to describing properties of heavy flavor systems (see, e.g., Ref. [59]). However, on one hand, it is challenging to well define the chiral condensate due to the quadratic divergence brought by the current quark mass term [15,60-62]. On the other hand, it is, in principle, difficult to separate the DCSB effect from the explicit mass scale arising from the ECSB term. To solve these problems, one needs to determine appropriately the quark condensate in the case of both light flavor and heavy flavor quarks.

Different ways have been proposed to remove the quadratic divergence of the condensate beyond the chiral limit. For example, it has been proposed that, since the DSEs have multisolutions for light flavor quarks, the divergence 
can be canceled by taking the trace of the sum of different solutions [4-6]. However, at high temperature, high chemical potential, or large current quark mass, there might be only one solution and this method cannot be adapted there.

The most straightforward way is to eliminate the quadratic divergence by doing subtraction. One of the most commonly used schemes is to subtract a fraction of strange quark condensate from light quark condensate, where the fraction coefficient is carefully designed to remove the quadratic divergence $[18,27,49]$. Another scheme is to subtract the derivative of the condensate [35,47]. Both of these two schemes are successful in removing the quadratic divergence induced by the nonzero current quark mass. However, there is still a logarithmic divergence, which already exists at chiral limit but is not serious and gets quite significant in the case of heavy flavor quarks. The logarithmic divergence of the condensate can be, in principle, absorbed by the renormalization constant of quark propagator near chiral limit, which is generally represented by the Gell-Mann-Oakes-Renner (GOR) relation. However, the renormalization of the composite operator becomes more complicated for heavy quarks and needs to be carefully treated.

In Ref. [5], it has also been proposed that the condensate can be extracted from the ultraviolet behavior of the mass function. However, in case of a finite current quark mass, the ultraviolet contribution to the condensate is much smaller than that from the current quark mass, and the fitting process needs to be extremely careful.

Inspired by these earlier works, if one takes the first subtraction scheme mentioned above to eliminate the contribution of the current quark mass, and then fits the ultraviolet behavior of the condensate, we can extract the quark condensate from the quark propagator while avoiding both the logarithm and the quadratic divergences [35]. This process can be extended to large current quark mass since it does not require the existence of multisolutions of the DSEs; i.e., we verify a well-defined and divergence-free quark condensate beyond the chiral limit directly in terms of the dressed quark propagator.

In our calculation, the current quark mass dependence of the condensate reveals a critical mass in the neighborhood of $\Lambda_{\mathrm{QCD}}$ (roughly the s-quark current mass). The critical mass confirms the previous studies of parton distribution amplitude (PDA) of mesons, where a critical mass has also been found near the s-quark mass so that the respective PDA becomes asymptotic $[63,64]$. These studies indicate a transition boundary for internal hadron dynamics between the different mass generation mechanisms.

The remainder of this paper is organized as follows. In Sec. II, we describe the DSE approach and the setups. In Sec. III A, we describe the theoretical framework of extracting the condensate introduced herein. In Sec. III B, we present our numerical results and discussions. Section IV provides a summary and perspective.

\section{THE GAP EQUATION}

The quark condensate is the trace of the fully dressed quark propagator. The starting point is the renormalized quark propagator $S$, as schematically depicted in Fig. 1. It reads in the Dyson-Schwinger equation approach as

$$
S^{-1}(p)=Z_{2}\left(i \not p+Z_{m} m^{\zeta}\right)+\Sigma(p),
$$

with the self-energy

$\Sigma(p)=g^{2} Z_{1} \int_{q}^{\Lambda} D_{\mu \nu}(p-q) \frac{\lambda^{a}}{2} \gamma_{\mu} S(q) \frac{\lambda^{a}}{2} \Gamma_{\nu}(q, p)$,

where $Z_{1}, Z_{2}$, and $Z_{m}$ are the vertex, quark wave function, and mass renormalization constants, respectively. $m^{\zeta}$ is the renormalized current quark mass, $\lambda^{a}$ are the color matrices, $\int_{q}^{\Lambda}$ represents a Poincaré invariant regularization of the fourdimensional integral, with $\Lambda$ as the ultraviolet regularization mass scale. $\Gamma_{\nu}$ and $D_{\mu \nu}$ are the dressed quark-gluon vertex and the dressed gluon propagator, respectively.

From the above equations, we can see that the propagator $S$ depends on the dressed gluon propagator $D_{\mu \nu}$ and the dressed quark-gluon vertex $\Gamma_{\nu}$ explicitly, which must be specified. Instead of solving the coupled quark and ghost and gluon system, one may rather choose to employ some suitable ansatz for the coupling and interaction in Eq. (2), which has sufficient integrated strength in the infrared to achieve the dynamical mass generation mechanism.

For the quark-gluon vertex, we apply the rainbow vertex truncation $\Gamma_{\mu}(q, p)=\gamma_{\mu}$. Under this truncation, however, the DSE solutions are not multiplicatively renormalizable and depend on the chosen renormalization point, unless the model for the gluon propagator is carefully designed.

A commonly used ansatz in practical calculation is to model the $g^{2} D_{\mu \nu}(k)$. In order to obtain a multiplicative renormalizability (MR), we take the renormalization procedure described explicitly in Appendix A of Ref. [49].

First, we replace the dressed quark-gluon vertex $\Gamma_{\mu}$ with the renormalized bare one, i.e.,

$$
\Gamma_{\mu}(q, p)=Z_{1} \gamma_{\mu} .
$$

This leaves the MR intact, because $Z_{1}$ renormalizes similar to the dressed vertex $\Gamma_{\mu}$. Now we have a factor $Z_{1}^{2}$ in front

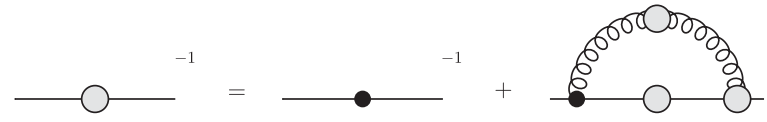

FIG. 1. The Feynman diagram for the Dyson-Schwinger equation of the quark propagator in Eq. (1). The solid line with gray circle denotes the dressed quark propagator, the solid line with black circle denotes the bare quark propagator, the curly line with gray circle denotes the dressed gluon propagator, and the black and gray circles stand for the bare vertex and the dressed vertex, respectively. 
of the self-energy integral (2). Second, we make use of the Slavnov-Taylor identity and replace

$$
Z_{1}^{2}=\left(Z_{2} \tilde{Z}_{1} / \tilde{Z}_{3}\right)^{2}
$$

where $\tilde{Z}_{1}$ is the renormalization factor of the ghost-gluon vertex and $\tilde{Z}_{3}$ is the renormalization of the ghost. Again, this leaves the MR intact. To take a model for the interaction, we do the replacement

$$
Z_{2}^{2}\left(\frac{\tilde{Z}_{1}}{\tilde{Z}_{3}}\right)^{2} g^{2} D_{\mu \nu}(k)=Z_{2}^{2} k^{2} \mathcal{G}\left(k^{2}\right) D_{\mu \nu}^{\mathrm{free}}(k)
$$

where $D_{\mu \nu}^{\text {free }}(k)=\left(\delta_{\mu \nu}-\frac{k_{\mu} k_{\nu}}{k^{2}}\right) \frac{1}{k^{2}}$ is the Landau-gauge-free gluon propagator, and $\mathcal{G}\left(k^{2}\right)$ is the effective interaction, which can be represented with models.

The interaction model $\mathcal{G}\left(k^{2}\right)$ is usually written as

$$
k^{2} \mathcal{G}\left(k^{2}\right)=k^{2} \mathcal{G}_{\mathrm{IR}}\left(k^{2}\right)+4 \pi \tilde{\alpha}_{\mathrm{pQCD}}\left(k^{2}\right)
$$

where $\tilde{\alpha}_{\mathrm{pQCD}}\left(k^{2}\right)$ is a bounded and monotonically decreasing regular continuation of the perturbative QCD running coupling to all values of spacelike $k^{2}$, and $\mathcal{G}_{\mathrm{IR}}\left(k^{2}\right)$ is the interaction at the infrared region and dominates in the region $|k|<\Lambda_{\mathrm{QCD}}$. The form of $\mathcal{G}_{\mathrm{IR}}\left(k^{2}\right)$ determines whether the DCSB and/or confinement can be realized.

In this work we adopt the Qin-Chang model [65], and the interaction is expressed as $\left(s=k^{2}\right)$

$$
\mathcal{G}(s)=\frac{8 \pi^{2}}{\omega^{4}} D e^{-s / \omega^{2}}+\frac{8 \pi^{2} \gamma_{m} \mathcal{F}(s)}{\ln \left[\tau+\left(1+s / \Lambda_{\mathrm{QCD}}^{2}\right)^{2}\right]},
$$

where $\mathcal{F}(s)=\left(1-e^{-s / 4 m_{t}^{2}}\right) / s$ with $m_{t}=0.5 \mathrm{GeV}, \gamma_{m}=$ $12 /\left(33-2 N_{f}\right)$ is the dimension anomaly with the flavor number $N_{f}$, and $\tau=e^{2}-1$ is a constant. Following Ref. [66], we take $N_{f}=4$ and $\Lambda_{\mathrm{QCD}}=0.234 \mathrm{GeV}$. The parameters $D$ and $\omega$ control the strength and the width of the interaction, respectively. In fact, observable properties of light-quark, ground-state, vector- and isospin-nonzero pseudoscalar mesons are insensitive to variations of $\omega \in[0.4,0.6] \mathrm{GeV}$, as long as

$$
\varsigma^{3}:=D \omega=\text { constant }
$$

In this work, following the commonly used values, we set $D=1.024 \mathrm{GeV}^{2}$ and $\omega=0.5 \mathrm{GeV}$ [67].

After the vertex and gluon is specified, we can solve the equation for the dressed quark propagator, which can be decomposed as

$$
S^{-1}(p)=i \not p A\left(p^{2}\right)+B\left(p^{2}\right) .
$$

The renormalization condition of this approach reads

$$
\begin{aligned}
\left.A\left(p^{2}\right)\right|_{p^{2}=\zeta^{2}, m^{\zeta}=0} & =1, \\
\left.\frac{\partial B\left(p^{2}\right)}{\partial m^{\zeta}}\right|_{p^{2}=\zeta^{2}, m^{\zeta}=0} & =1,
\end{aligned}
$$

where $\zeta$ is again the renormalization point and $m^{\zeta}$ is the renormalized current quark mass. The dressed quark mass function is defined as

$$
M\left(p^{2}\right)=B\left(p^{2}, \zeta^{2}\right) / A\left(p^{2}, \zeta^{2}\right)
$$

which is independent of the renormalization point $\zeta$.

In this paper, we adopt two renormalization points, $\zeta=19 \mathrm{GeV}$ and $\zeta=\infty$, for different approaches of calculating the quark condensate. For $\zeta=19 \mathrm{GeV}$, the renormalization constants $Z_{2}$ and $Z_{m}$ are determined using Eq. (7), and $Z_{1}=Z_{2}^{2}$ as our assumption. For $\zeta=\infty$, all the renormalization constants are set to unity.

\section{RESULTS AND DISCUSSIONS}

\section{A. Quark condensate beyond chiral limit}

Now applying the conventional definition of the chiral condensate as the trace of the quark propagator, one can compute the condensate via

$$
\begin{aligned}
\operatorname{Tr}[S] & =-Z_{4} N_{c} N_{f} \int_{k}^{\Lambda} \operatorname{tr}_{\mathrm{D}}[S(k, \zeta)] \\
& =-\frac{Z_{4} N_{c} N_{f}}{2 \pi^{2}} \int_{0}^{\Lambda} \frac{k^{3} \mathrm{~d} k M\left(k^{2}, \zeta^{2}\right) / A\left(k^{2}, \zeta^{2}\right)}{k^{2}+M^{2}\left(k^{2}, \zeta^{2}\right)},
\end{aligned}
$$

where $Z_{4}=Z_{2} Z_{m}$. If the current quark mass $m^{\zeta}$ has a finite value, then the constituent quark mass $M\left(p^{2}, \zeta^{2}\right)$ does not vanish at large $p^{2}$, and the above integration suffers from quadratic divergence.

In Refs. [15,47], the condensate beyond the chiral limit is defined by a subtraction

$$
-\langle\bar{q} q\rangle_{m^{\zeta}}:=\left(1-m^{\zeta} \frac{\partial}{\partial m_{\zeta}}\right) \operatorname{Tr}[S] .
$$

For simplicity, in the following, the condensate calculated using Eqs. (9) and (10) will be referred to as "condensate A," and the approach to calculate condensate A will be referred to as "scheme A." In this paper, for condensate A, the renormalization point is chosen as $\zeta=19 \mathrm{GeV}$.

At chiral limit and small current quark mass, scheme A works fine in calculating the quark condensate. However, at large current quark mass, the condensate A still suffers from a loglike divergence. In Fig. 2, we presented the calculated 


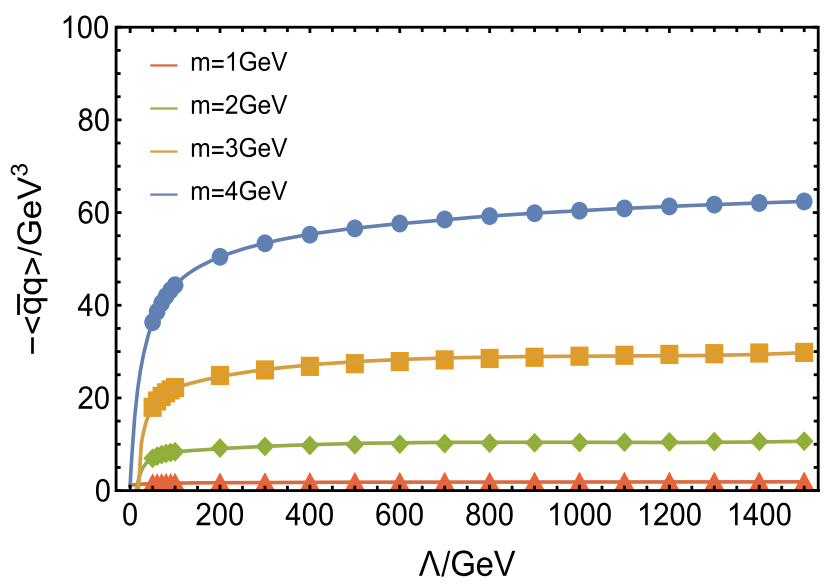

FIG. 2. The calculated quark condensate using Eq. (9), as a function of the cutoff $\Lambda$. Different lines correspond to different current quark masses. The renormalization point is $\zeta=19 \mathrm{GeV}$.

result of condensate A as a function of ultraviolet cutoff $\Lambda$ at several large current quark masses. From the figure, it can be seen that, for large quark mass, the logarithmic dependence of the cutoff still exists. As mentioned above, the renormalization of such a composite operator is constrained by the GOR relation at the chiral limit; however, the cutoff dependence shown in Fig. 2 suggests that it may fail for large quark mass, and the log divergence needs to be carefully treated. Note that the pion condensate does not suffer from this problem since it can be constrained by the axial-vector Ward identity for any quark mass $[68,69]$.

In order to obtain a better behavior of the quark condensate at large current quark mass, in this paper, we extract the condensate directly by fitting the asymptotic behavior of the composite operator.

According to the operator product expansion technique, the asymptotic behavior of the mass function at large momentum is $[5,36]$

$$
\begin{aligned}
M\left(p^{2}\right)_{\text {asym }}= & \frac{\mathcal{C}}{p^{2}}\left[\frac{1}{2} \ln \left(p^{2} / \Lambda_{\mathrm{QCD}}^{2}\right)\right]^{\gamma_{m}-1} \\
& +\hat{m}\left[\ln \left(p^{2} / \Lambda_{\mathrm{QCD}}^{2}\right)\right]^{-\gamma_{m}},
\end{aligned}
$$

where

$$
\mathcal{C}=\frac{-2 \pi^{2} \gamma_{m}}{3} \frac{\langle\bar{q} q\rangle^{\zeta 0}}{\left[\frac{1}{2} \ln \left(\zeta^{2} / \Lambda_{\mathrm{QCD}}^{2}\right)\right]^{\gamma_{m}}}
$$

and

$$
\hat{m}=m^{\zeta}\left[\frac{1}{2} \ln \left(\zeta^{2} / \Lambda_{\mathrm{QCD}}^{2}\right)\right]^{\gamma_{m}} .
$$

Both $\mathcal{C}$ and $\hat{m}$ are renormalization-independent quantities. $\langle\bar{q} q\rangle^{\zeta 0}$ in Eq. (12) is the condensate at chiral limit with renormalization point $\zeta$.
By inserting Eq. (11) into Eq. (9), we have

$$
\begin{aligned}
\operatorname{Tr}\left[S_{\text {asym }}\right]= & -\langle\bar{q} q\rangle^{\zeta 0} \frac{\left[\ln \left(\Lambda^{2} / \Lambda_{\mathrm{QCD}}^{2}\right)\right]^{\gamma_{m}}}{\left[\ln \left(\zeta^{2} / \Lambda_{\mathrm{QCD}}^{2}\right)\right]^{\gamma_{m}}} \\
& +\frac{N_{c}}{2 \pi^{2}} \int^{\Lambda} p \hat{m}\left[\ln \left(p^{2} / \Lambda_{\mathrm{QCD}}^{2}\right)\right]^{-\gamma_{m}} \mathrm{~d} p .
\end{aligned}
$$

It is apparent that there exists a logarithmic divergence when $m^{\zeta}=0$ and an extra quadratic divergence for finite $m^{\zeta}$.

From Eq. (14), one can notice that the trace of the quark propagator is cutoff dependent and needs a subtraction to eliminate the quadratic divergence that comes from the linear dependence of the current quark mass in the mass function and then a renormalization to deal with the logarithmic divergence.

In the ordinary approach, one would apply renormalization first and then do the subtraction. In this paper, we present an approach that does the subtraction first and then extracts the condensate from the logarithmic divergence behavior.

Applying Eq. (10) on Eq. (14), we find that the second term in Eq. (14) vanishes. However, the logarithmic divergence remains as

$$
\left(1-m^{\zeta} \frac{\partial}{\partial m^{\zeta}}\right) \operatorname{Tr}\left[S_{\text {asym }}\right]=-\langle\bar{q} q\rangle\left[\frac{1}{2} \ln \left(\Lambda^{2} / \Lambda_{\mathrm{QCD}}^{2}\right)\right]^{\gamma_{m}} .
$$

Notice that $\langle\bar{q} q\rangle$ defined in the above equation is a renormalization and cutoff-independent condensate. We rewrite the rhs of Eq. (15) as

$$
\mathcal{F}\left(\langle\bar{q} q\rangle, \Lambda_{\mathrm{QCD}}, \gamma_{m}\right)=-\langle\bar{q} q\rangle\left[\frac{1}{2} \ln \left(\Lambda^{2} / \Lambda_{\mathrm{QCD}}^{2}\right)\right]^{\gamma_{m}} .
$$

After figuring out the asymptotic behavior of the subtracted condensate, we can use $\mathcal{F}\left(\langle\bar{q} q\rangle, \Lambda_{\mathrm{QCD}}, \gamma_{m}\right)$ defined in Eq. (16) to fit the trace of the propagator, $\left(1-m^{\zeta} \frac{\partial}{\partial m_{\zeta}}\right) \operatorname{Tr}\left[S_{\text {asym }}\right]$, and we can extract the condensate $\langle\bar{q} q\rangle$ as the fitting parameter. For simplicity, we do the renormalization at infinity, i.e., taking $\zeta=\Lambda$, and sending $\Lambda$ to infinity. This is equivalent to the unrenormalized approach and all the renormalization constants become 1 . The renormalized condensate at renormalization point $\zeta$ can be derived as

$$
\langle\bar{q} q\rangle_{\zeta}=\langle\bar{q} q\rangle\left[\frac{1}{2} \ln \left(\zeta^{2} / \Lambda_{\mathrm{QCD}}^{2}\right)\right]^{\gamma_{m}}
$$

For simplicity, in the following, the condensate calculated from extracting asymptotic behavior will be referred to as "condensate B," and the approach to calculate condensate B will be referred to as "scheme B." 


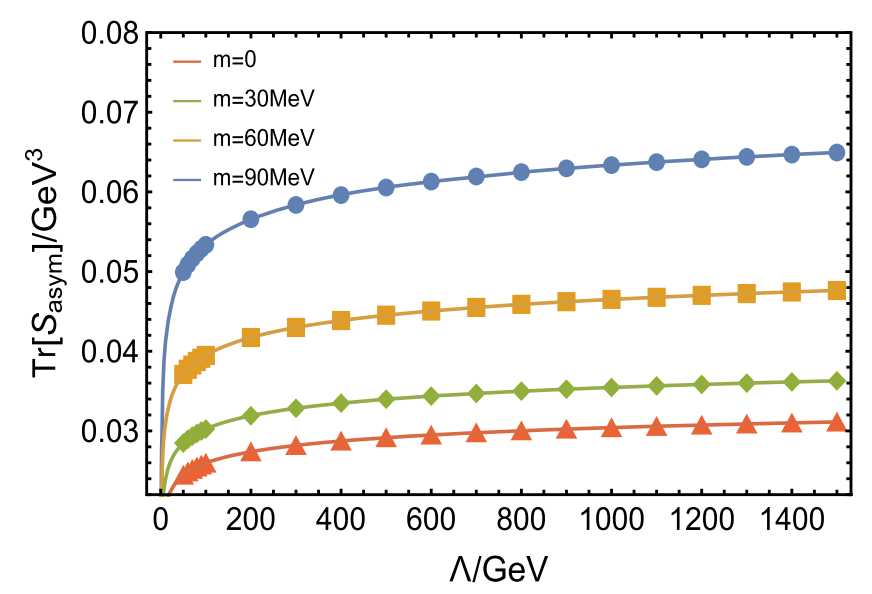

FIG. 3. The calculated trace of the quark propagator as a function of cutoff $\Lambda$. The symbols with different shapes correspond to the trace of the propagator calculated from Eq. (9), and the solid lines with different colors correspond to the fitted curves in Eq. (16).

The result of the fitting is shown in Fig. 3. As can be seen from the figure, the fit is with a quite high precision for a range of current quark masses.

In the chiral limit, the fitting parameters are $\Lambda_{\mathrm{QCD}}=$ $0.2749 \mathrm{GeV}, \gamma_{m}=0.4799$, and $-\langle\bar{q} q\rangle^{0}=(223 \mathrm{MeV})^{3}$. The condensate at $2 \mathrm{GeV}$ is derived from Eq. (17): $-\langle\bar{q} q\rangle_{\zeta=2 \mathrm{GeV}}^{0}=(249 \mathrm{MeV})^{3}$. This is comparable with lattice studies [see, e.g., $(252(5)(10) \mathrm{MeV})^{3}$ in Ref. [70] and other lattice results in Refs. [71-77] ].

We need to mention that, although both Figs. 2 and 3 show a logarithmic dependence on the cutoff, they have totally different meanings. In Fig. 2, this divergence is what we intend to avoid since we have already applied the renormalization. In Fig. 3, however, this divergence is exactly what we need in order to extract the quark condensate.

Now we get a cutoff-independent chiral condensate in terms of the quark propagator beyond the chiral limit. In Fig. 4, we present the obtained result of the quark condensate as a function of the current quark mass and the comparison of the two schemes. From the figure, we can see that the two approaches agree well with each other at chiral condensate and small current quark mass. For large current quark mass, however, the two schemes give different results. There are two key differences that should be noticed. First, the dashed lines, which are the condensates calculated using Eqs. (9) and (10), exhibit a significant cutoff dependence, especially at large current quark masses. This is the same as what we can see from Fig. 2, where the logarithmic divergence of the condensate is explicit. The solid lines are the condensate extracted from the asymptotic behavior. For larger quark mass, there is slight $\Lambda$ dependence that comes from the error of the fitting process. The error increases slightly due to the sizable current quark mass scale that drives the ultraviolet behavior of quark mass function to deviate from the

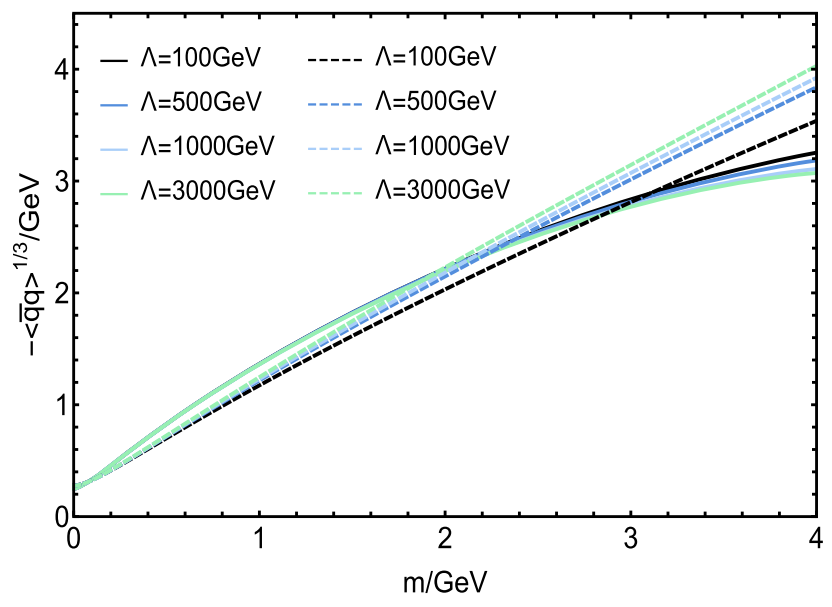

FIG. 4. The calculated current quark mass dependence of the quark condensate with different cutoffs. The dashed lines correspond to the condensate calculated using Eqs. (9) and (10) and are the counterparts of Fig. 2. The solid lines correspond to the condensate extracted from the asymptotic behavior of the quark propagator trace. For each method, we calculate the condensate under several different values of the cutoff $\Lambda$.

asymptotic form. The error can be reduced by setting a larger cutoff.

Another difference is the behavior at large current quark mass. For the dashed lines, the condensate depends almost linearly on the current quark mass, while for the solid lines, the increasing speed slows down obviously at large $m^{\zeta}$. The rapid increases of condensate $\mathrm{A}$ is mainly due to the remnant of the logarithmic divergence. However, it does not change the conclusion about the existence of the critical mass as will be shown below.

\section{B. Interference between DCSB and ECSB}

From Fig. 4, we find that the chiral condensate increases monotonically with the current quark mass. This behavior seemingly means that the DCSB effect is stronger for heavier quarks. This is in contradiction to the nonrelativistic QCD (NRQCD) computation of heavy quarkonium, whose result shows that the heavier system can be better described with current quark by the nonrelativistic potential [78-80]. However, the increasing condensate only means that the mass scale brought by the chiral symmetry breaking effect grows along with the scale of current quark mass. This mass scale contains three parts of contribution: the dynamical part (DCSB), the explicit part (ECSB), and their interference. By doing the subtraction, the ECSB effect is excluded from the definition of condensate, and the condensate consists of the DCSB and the interference part. The sum of the DCSB and the interference effect increases as the ECSB increases, but the proportion from the DCSB effect should be small when the current quark mass is large, since the interaction is negligible for extremely heavy quark systems. To illustrate this, we can define a dimensionless chiral susceptibility as 


$$
\chi(m)=\frac{-\partial\langle\bar{q} q\rangle^{1 / 3}}{\partial m} .
$$

The calculated current quark mass dependence of the dimensionless chiral susceptibility is shown in Fig. 5. The response of the quark condensate to the current mass increases near the chiral limit and then decreases gradually. There then exists a critical mass, $m_{\text {crit }}=0.22 \mathrm{GeV}$, which separates the two regions (in comparison, the critical mass obtained from condensate $\mathrm{A}$ is $m_{\text {crit }}=0.45 \mathrm{GeV}$ ). The critical mass is well located around $\Lambda_{\mathrm{QCD}} \sim 0.234 \mathrm{GeV}$, which is naturally the critical scale between the nonperturbative and perturbative dynamics. It also puts the strange quark on the transition boundary for internal hadron dynamics, since this critical scale is located in the neighborhood of the strange quark mass. This indicates that no expansions in the strange quark mass can be reliable. This critical mass represents the relation between the DCSB and ECSB effect through the interference. For $m<m_{\text {crit }}$, the $\chi(m)$ function is increasing, which indicates that the interference is mainly induced by the DCSB effect; while for $m>m_{\text {crit }}$, the $\chi(m)$ function is decreasing, indicating that the interference is mainly induced by the ECSB effect. Therefore, the $\chi(m)$ function can be taken to identify which effect dominates the interference.

Also, the behavior of $\chi(m)$ is surprisingly consistent with the analysis of the PDA of hadrons [63,64]. For the mesons with light quark, the PDA is a broadening function in comparison to the asymptotic form $6 x(1-x)$, while for heavy quarkonia, the PDA becomes narrower. The PDA of hadrons gives then this critical mass also in the neighborhood of the strange quark, where the respective PDA becomes precisely the asymptotic form. This consistence reveals the connection between the internal hadron dynamics and the condensate of quarks inside. The different response of the quark condensate to the external source might induce an intrinsic transition of internal hadron dynamics.

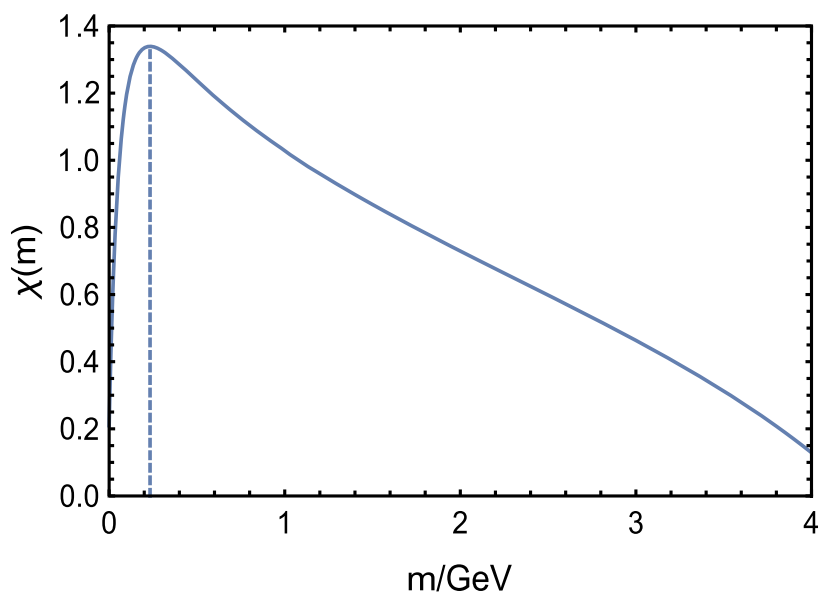

FIG. 5. The calculated dimensionless chiral susceptibility $\chi(m)$, where the dashed vertical line indicates the steepest descent mass of the corresponding condensate $-\langle\bar{q} q\rangle^{1 / 3}$.
Notice that the condensate defined above is purely in terms of the quark propagator and is thus independent of hadrons. It is then interesting to investigate the behavior of the condensate when putting the quarks into hadrons. In considering the pion, these features are expressed in the axialvector Ward-Takahashi identity (AV-WTI) [42,68,81]. Equating pole contributions of the corresponding axial and pseudoscalar vertexes in the AV-WTI, it entails

$$
f_{\pi}^{2} m_{\pi}^{2}=\left(m_{u}^{\zeta}+m_{d}^{\zeta}\right) f_{\pi} \rho_{\pi}^{\zeta}
$$

where $f_{\pi}$ and $\rho_{\pi}^{\zeta}$ are defined as

$$
\begin{aligned}
i f_{\pi} P_{\mu} & =\left\langle 0\left|\bar{q} \gamma_{5} \gamma_{\mu} q\right| \Pi\right\rangle \\
& =Z_{2}(\zeta, \Lambda) \operatorname{tr}_{\mathrm{D}} \int_{k}^{\Lambda} i \gamma_{5} \gamma_{\mu} S\left(k_{+}\right) \Gamma_{\pi}(k ; P) S\left(k_{-}\right), \\
i \rho_{\pi}^{\zeta} & =-\left\langle 0\left|\bar{q} i \gamma_{5} q\right| \Pi\right\rangle \\
& =Z_{4}(\zeta, \Lambda) \operatorname{tr}_{\mathrm{D}} \int_{k}^{\Lambda} \gamma_{5} S\left(k_{+}\right) \Gamma_{\pi}(k ; P) S\left(k_{-}\right),
\end{aligned}
$$

respectively, where $\Gamma_{\pi}(k ; P)$ is the pion Bethe-Salpeter amplitude, which reads

$$
\Gamma_{\pi}(k ; P)=\int_{q}^{\Lambda} S\left(q_{+}\right) \Gamma_{\pi}(q ; P) S\left(q_{-}\right) K(q, k ; P),
$$

where $K(q, k ; P)$ is the fully amputated quark-antiquark scattering kernel and $P^{2}=-m_{\pi}^{2}, k_{ \pm}=k \pm P / 2$, and $q_{ \pm}=q \pm P / 2$, without loss of generality in a Poincaré covariant approach.

The relation connects the pion mass $m_{\pi}$ and decay constant $f_{\pi}$ to the $u$ and $d$ quark current masses and $\kappa_{\pi}^{\zeta}:=f_{\pi} \rho_{\pi}^{\zeta}$, namely the in-hadron condensate introduced in Refs. $[60,61,69]$. The identity holds in the whole range of the current quark mass.

Using QCD quark-level Goldberger-Treiman relations, one can prove [68]

$$
f_{\pi}^{0} \rho_{\pi}^{\zeta 0}=-\langle\bar{q} q\rangle^{\zeta 0},
$$

where the superscript 0 indicates that the quantity is computed in the chiral limit. The relation means that the two definitions of the quark condensate coincide at the chiral limit. However, the in-hadron condensate could deviate against the quark condensate, since the quarks are now in the bound state and the interaction could induce a change of the DCSB effect of quarks. To identify such a point explicitly, we define the ratio of the two condensates as

$$
r_{h}(m):=\frac{f_{\pi} \rho_{\pi}}{-\langle\bar{q} q\rangle} .
$$

The calculated current mass dependence of the ratio $r_{h}(m)$ is illustrated in Fig. 6. As we can see in Fig. 6, in 


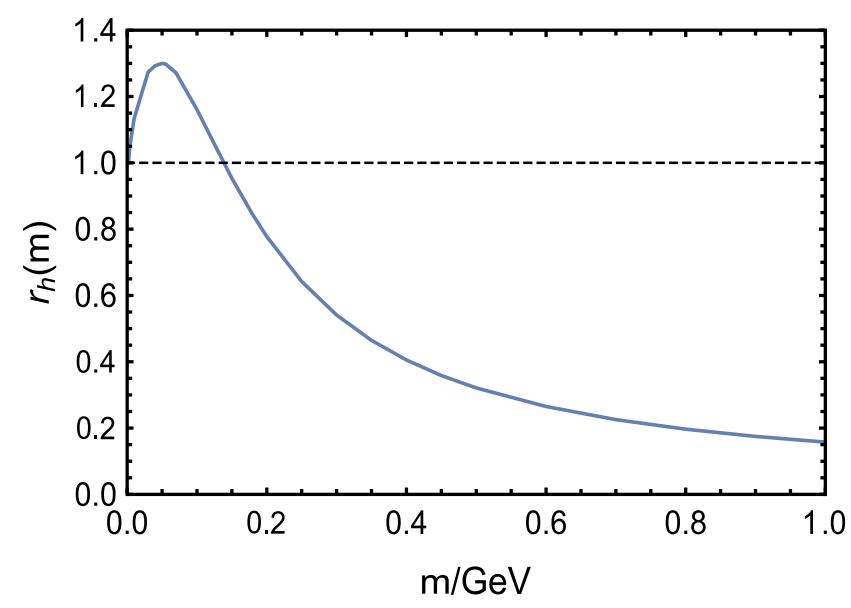

FIG. 6. The computed ratio $r_{h}(m)$ defined in Eq. (24), where the dashed horizontal line indicates $r_{h}(m)=1$.

sufficiently small current masses with $r_{h}>1$, the in-hadron condensate is larger than the quark condensate, which means that the interaction enhances the DCSB effect of quarks. Again, we can find a critical mass in the neighborhood of the strange quark, $m=0.13 \mathrm{GeV}$ (in comparison, the critical mass obtained from condensate $\mathrm{A}$ is $m=0.09 \mathrm{GeV}$ ); after that, the ratio is smaller than 1 where the in-hadron condensate starts to be smaller than the quark condensate. In this region, the interaction in bound states reduces the DCSB effect and leads the system gradually to the perturbative region.

The similarity of the variation behavior of the $r_{h}(m)$ with the temperature dependence of the trace anomaly $(\varepsilon-3 P) / T^{4}$, which plays the role of a measure of the interaction energy stored in the system (see, e.g., Refs. [44,82-84]), confirms this point of view definitely.

This again illustrates the transition of the internal hadron dynamics, and moreover, it manifests that this transition is induced by the interference between the DCSB and ECSB effects of the quark.

\section{SUMMARY AND REMARKS}

In summary, we have shown that a cutoff-independent condensate can be extracted after analyzing the asymptotic behavior of the quark mass function. This verifies a well-defined quark condensate at the level of quark propagator beyond the chiral limit. Along with the increase of current quark mass, the DCSB effect manifested via the condensate also shows an increasing behavior because of the interference of the mass scale between the two mass generation mechanisms. The relative contribution can be evaluated after defining a dimensionless chiral susceptibility. It gives then a critical mass around $\Lambda_{\mathrm{QCD}}, m=0.22 \mathrm{GeV}$. In the case where the current quark mass is smaller, the ECSB effect enhances the DCSB effect. For heavier quarks, the ECSB effect reduces the DCSB effect, which is then consistent with the NRQCD computations of heavy quarkonia.

Moreover, as we mentioned above, this condensate is independent of hadrons and then different from the inhadron condensate. This difference represents the effect of the interaction in hadrons. In fact, again we find a critical mass close to the value from dimensionless chiral susceptibility, $m=0.13 \mathrm{GeV}$. For lighter quarks, as indicated by the dimensionless chiral susceptibility, the external source enhances the DCSB effect, and consequently, the interaction in hadrons brings in a stronger DCSB effect. When the quark mass exceeds the critical mass, the in-hadron condensate is then smaller and thus the DCSB effect is reduced by the inside interaction of hadrons.

These observations are surprisingly consistent with the previous studies of the PDA of hadrons. As the current quark mass increases, the PDA of mesons with equal quark mass changes from a broadening function to a narrow peak, and there exists then a critical mass also in the neighborhood of the strange quark, where the respective PDA becomes precisely the asymptotic form $6 x(1-x)$. All these phenomena reveal that an intrinsic transition of internal hadron dynamics takes place, and more interestingly, there could be signals from the experiments of strange matter since the location of the transition is very close.

\section{ACKNOWLEDGMENTS}

The work was supported by the National Natural Science Foundation of China under Contracts No. 11435001 and No. 11775041. F. G. is grateful for the support from the Alexander von Humboldt Foundation.
[1] C. D. Roberts, D. G. Richards, T. Horn, and L. Chang, Prog. Part. Nucl. Phys. 120, 103883 (2021).

[2] F. T. Hawes, C. D. Roberts, and A. G. Williams, Phys. Rev. D 49, 4683 (1994).

[3] H. J. Munczek, Phys. Rev. D 52, 4736 (1995).

[4] L. Chang, Y.X. Liu, M. S. Bhagwat, C. D. Roberts, and S. V. Wright, Phys. Rev. C 75, 015201 (2007).
[5] R. Williams, C. S. Fischer, and M. R. Pennington, Phys. Lett. B 645, 167 (2007).

[6] H. Chen, W. Yuan, and Y. X. Liu, J. Phys. G 36, 064073 (2009); K. L. Wang, S. X. Qin, Y. X. Liu, L. Chang, C. D. Roberts, and S. M. Schmidt, Phys. Rev. D 86, 114001 (2012).

[7] A. C. Aguilar and J. Papavassiliou, Phys. Rev. D 83, 014013 (2011). 
[8] M. Mitter, J. M. Pawlowski, and N. Strodthoff, Phys. Rev. D 91, 054035 (2015).

[9] J. Braun, L. Fister, J. M. Pawlowski, and F. Rennecke, Phys. Rev. D 94, 034016 (2016).

[10] A. Abhishek and H. Mishra, Phys. Rev. D 99, 054016 (2019).

[11] C. D. Roberts, Symmetry 12, 1468 (2020).

[12] S. Descotes-Genon, N. H. Fuchs, L. Girlanda, and J. Stern, Eur. Phys. J. C 34, 201 (2004).

[13] C. S. Fischer, J. Luecker, and J. A. Mueller, Phys. Lett. B 702, 438 (2011).

[14] S. J. Brodsky, C. D. Roberts, R. Shrock, and P. C. Tandy, Phys. Rev. C 85, 065202 (2012).

[15] C. McNeile, A. Bazavov, C. T. H. Davies, R. J. Dowdall, K. Hornbostel, G. P. Lepage, and H. D. Trottier, Phys. Rev. D 87, 034503 (2013).

[16] Z. F. Cui, F. Y. Hou Y. M. Shi, Y. L. Wang, and H. S. Zong, Ann. Phys. (Amsterdam) 358, 172 (2015).

[17] H. Raval and B. P. Mandala, Nucl. Phys. B946, 114699 (2019).

[18] J. Braun, W. J. Fu, J. M. Pawlowski, F. Rennecke, D. Rosenblüh, and S. Yin, Phys. Rev. D 102, 056010 (2020).

[19] F. Gao and J. M. Pawlowski, Phys. Rev. D 102, 034027 (2020).

[20] Y.Z. Xu, C. Shi, X. T. He, and H. S. Zong, Phys. Rev. D 102, 114011 (2020).

[21] Z. Bai, L. Chang, J. Y. Chao, F. Gao, and Y. X. Liu, Phys. Rev. D 104, 014005 (2021).

[22] P. J. Gunkel and C. S. Fischer, Eur. Phys. J. A 57, 147 (2021).

[23] L. Chang, Y. X. Liu, and H. Guo, Phys. Rev. D 72, 094023 (2005); J. H. Huang, T. T. Sun, and H. Chen, Phys. Rev. D 101, 054007 (2020).

[24] W. Yuan and Y. X. Liu, arXiv:gr-qc/0610114; S. J. Brodsky and R. Shrock, Proc. Natl. Acad. Sci. U.S.A. 108, 45 (2011).

[25] P. Isserstedt, C. S. Fischer, and T. Steinert, Phys. Rev. D 103, 054012 (2021).

[26] A. Bazavov, H. T. Ding, P. Hegde, O. Kaczmarek, F. Karsch, E. Laermann, S. Mukherjee, P. Petreczky, C. Schmidt, D. Smith, W. Soeldner, and M. Wagner, Phys. Rev. Lett. 109, 192302 (2012); A. Bazavov, H. T. Ding, P. Hegde, O. Kaczmarek, F. Karsch, E. Laermann et al., Phys. Rev. D 95, 054504 (2017); A. Bazavov, H. T. Ding, P. Hegde, O. Kaczmarek, F. Karsch, E. Laermann et al. (HotQCD Collaboration), Phys. Rev. D 96, 074510 (2017); A. Bazavov et al. (HotQCD Collaboration), Phys. Lett. B 795, 15 (2019).

[27] A. Bazavov, T. Bhattacharya, M. Cheng, C. DeTar, H. T. Ding, S. Gottlieb et al., Phys. Rev. D 85, 054503 (2012).

[28] S. Borsanyi, Z. Fodor, S. D. Katz, S. Krieg, C. Ratti, and K. K. Szabo, Phys. Rev. Lett. 111, 062005 (2013); C. Bonati, M. D'Elia, M. Mariti, M. Mesiti, F. Negro, and F. Sanfilippo, Phys. Rev. D 92, 054503 (2015); R. Bellwied, S. Borsanyi, Z. Fodor, J. Guenther, S. D. Katz, C. Ratti, and K. K. Szabo, Phys. Lett. B 751, 559 (2015).

[29] S. Borsanyi, Z. Fodor, J. N. Guenther, S. K. Katz, K. K. Szabo, A. Pasztor, I. Portillo, and C. Ratti, J. High Energy Phys. 10 (2018) 205; H. T. Ding, P. Hegde, O. Kaczmarek, F. Karsch, A. Lahiri, S. T. Li, S. Mukherjee, H. Ohno,
P. Petreczky, C. Schmidt, and P. Steinbrecher, Phys. Rev. Lett. 123, 062002 (2019).

[30] J. Braun, L. M. Haas, F. Marhauser, and J. M. Pawlowski, Phys. Rev. Lett. 106, 022002 (2011); A. K. Cyrol, M. Mitter, J. M. Pawlowski, and N. Strodthoff, Phys. Rev. D 97, 054006 (2018).

[31] W. J. Fu, J. M. Pawlowski, F. Rennecke, and B. J. Schaefer, Phys. Rev. D 94, 116020 (2016).

[32] F. Rennecke and B. J. Schaefer, Phys. Rev. D 96, 016009 (2017).

[33] X. Li, W. J. Fu, and Y. X. Liu, Phys. Rev. D 99, 074029 (2019).

[34] W. J. Fu, J. M. Pawlowski, and F. Rennecke, Phys. Rev. D 101, 054032 (2020).

[35] F. Gao, J. Papavassiliou, and J. M. Pawlowski, Phys. Rev. D 103, 094013 (2021).

[36] C. D. Roberts and A. G. Williams, Prog. Part. Nucl. Phys. 33, 477 (1994).

[37] C. D. Roberts and S. Schmidt, Prog. Part. Nucl. Phys. 45, S1 (2000); A. Bashir, L. Chang, I. C. Cloet, B. El-Bennich, Y. X. Liu, C. D. Roberts, and P. C. Tandy, Commun. Theor. Phys. 58, 79 (2012).

[38] R. Alkofer and L. von Smekal, Phys. Rep. 353, 281 (2001).

[39] C. S. Fischer, J. Phys. G 32, R253 (2006); C. S. Fischer, A. Maas, and J. M. Pawlowski, Ann. Phys. (Amsterdam) 324, 2408 (2009).

[40] A. Bender, D. Blaschke, Y. Kalinovsky, and C. D. Roberts, Phys. Rev. Lett. 77, 3724 (1996).

[41] L. Chang, Y. X. Liu, and C. D. Roberts, Phys. Rev. Lett. 106, 072001 (2011).

[42] S. X. Qin, L. Chang, Y. X. Liu, C. D. Roberts, and S. M. Schmidt, Phys. Lett. B 722, 384 (2013).

[43] D. Binosi, L. Chang, J. Papavassiliou, and C. D. Roberts, Phys. Lett. B 742, 183 (2015); D. Binosi, L. Chang, J. Papavassiliou, S. X. Qin, and C. D. Roberts, Phys. Rev. D 93, 096010 (2016); A. C. Aguilar, J. C. Cardona, M. N. Ferreira, and J. Papavassiliou, Phys. Rev. D 98, 014002 (2018).

[44] F. Gao, J. Chen, Y. X. Liu, S. X. Qin, C. D. Roberts, and S. M. Schmidt, Phys. Rev. D 93, 094019 (2016).

[45] S. X. Qin, L. Chang, Y. X. Liu, and C. D. Roberts, Phys. Rev. D 84, 014017 (2011); S. X. Qin and D. H. Rischke, Phys. Rev. D 88, 056007 (2013); S. X. Qin and C. D. Roberts, Chin. Phys. Lett. 38, 071201 (2021); L. F. Chen, S. X. Qin, and Y. X. Liu, Phys. Rev. D 102, 054015 (2020).

[46] C. S. Fischer and J. Luecker, Phys. Lett. B 718, 1036 (2013); C. S. Fischer, L. Fister, J. Luecker, and J. M. Pawlowski, Phys. Lett. B 732, 273 (2014).

[47] F. Gao and Y. X. Liu, Phys. Rev. D 94, 076009 (2016).

[48] C. Tang, F. Gao, and Y. X. Liu, Phys. Rev. D 100, 056001 (2019).

[49] C. S. Fishcer, Prog. Part. Nucl. Phys. 105, 1 (2019).

[50] Y. Nambu and G. Jona-Lasinio, Phys. Rev. 122, 345 (1961); 124, 246 (1961).

[51] S. P. Klevansky, Rev. Mod. Phys. 64, 649 (1992).

[52] M. Buballa, Phys. Rep. 407, 205 (2005).

[53] C. Ratti, M. A. Thaler, and W. Weise, Phys. Rev. D 73, 014019 (2006); W. J. Fu, Z. Zhang, and Y. X. Liu, Phys. Rev. D 77, 014006 (2008). 
[54] B. J. Schaefer, J. M. Pawlowski, and J. Wambach, Phys. Rev. D 76, 074023 (2007).

[55] A. Zacchi, R. Stiele, and J. Schaffner-Bielich, Phys. Rev. D 92, 045022 (2015).

[56] A. C. Aguilar, Z. Ahmed, C. Aidala et al., Eur. Phys. J. A 55, 190 (2019).

[57] Z. F. Cui, M. Ding, F. Gao, K. Raya, D. Binosi, L. Chang, C. D. Roberts, J. Rodíguez-Quinteror, and S. M. Schmidt, Eur. Phys. J. A 57, 5 (2021).

[58] C. D. Roberts, arXiv:2101.08340.

[59] J. Chen, M. H. Ding, L. Chang, and Y. X. Liu, Phys. Rev. D 95, 016010 (2017).

[60] P. Maris and C. D. Roberts, Phys. Rev. C 56, 3369 (1997).

[61] S. J. Brodsky, C. D. Roberts, R. Shrock, and P. C. Tandy, Phys. Rev. C 82, 022201(R) (2010).

[62] K. Langfeld, H. Markum, R. Pullirsch, C. D. Roberts, and S. M. Schmidt, Phys. Rev. C 67, 065206 (2003).

[63] F. Gao, L. Chang, and Y. X. Liu, Phys. Lett. B 770, 551 (2017).

[64] M. Ding, F. Gao, L. Chang, Y. X. Liu, and C. D. Roberts, Phys. Lett. B 753, 330 (2016).

[65] S. X. Qin, L. Chang, Y. X. Liu, C. D. Roberts, and D. J. Wilson, Phys. Rev. C 84, 042202(R) (2011).

[66] Q. W. Wang, S. X. Qin, C. D. Roberts, and S. M. Schmidt, Phys. Rev. D 98, 054019 (2018).

[67] S. X. Qin, C. D. Roberts, and S. M. Schmidt, Phys. Rev. D 97, 114017 (2018).

[68] P. Maris, C. D. Roberts, and P. C. Tandy, Phys. Lett. B 420, 267 (1998).

[69] L. Chang, C. D. Roberts, and P. C. Tandy, Phys. Rev. C 85, 012201(R) (2012).

[70] S. Aoki et al. (JLQCD and TWQCD Collaborations), Phys. Lett. B 665, 294 (2008).

[71] H. Fukaya, S. Aoki, T. W. Chiu, S. Hashimoto, T. Kaneko, H. Matsufuru, J. Noaki, K. Ogawa, M. Okamoto, T. Onogi, and N. Yamada (JLQCD Collaboration), Phys. Rev. Lett. 98, 172001 (2007).

[72] H. Fukaya, S. Aoki, T. W. Chiu, S. Hashimoto, T. Kaneko, H. Matsufuru, J. Noaki, K. Ogawa, T. Onogi, and N. Yamada (JLQCD and TWQCD Collaborations), Phys. Rev. D 76, 054503 (2007).

[73] H. Fukaya, S. Aoki, S. Hashimoto, T. Kaneko, J. Noaki, T. Onogi, and N. Yamada, Phys. Rev. Lett. 104, 122002 (2010).

[74] J. Noaki et al. (JLQCD and TWQCD Collaborations), Proc. Sci. LAT2009 (2009) 096 [arXiv:0910.5532].

[75] T. W. Chiu, T. H. Hsieh, and P. K. Tseng (TWQCD Collaboration), Phys. Lett. B 671, 135 (2009).

[76] L. Giusti and M. Luscher, J. High Energy Phys. 03 (2009) 013.

[77] J. Noaki, S. Aoki, T. W. Chiu, H. Fukaya, S. Hashimoto, T. H. Hsieh, T. Kaneko, H. Matsufuru, T. Onogi, E. Shintani, and N. Yamada (JLQCD and TWQCD Collaborations), Phys. Rev. Lett. 101, 202004 (2008).

[78] G. T. Bodwin, E. Braaten, and G. P. Lepage, Phys. Rev. D 51, 1125 (1995); 55, 5853 (1997).

[79] J. Komijani, P. Petreczky, and J. H. Weber, Prog. Part. Nucl. Phys. 113, 103788 (2020).

[80] E. Eichten, K. Gottfried, T. Kinoshita, K. D. Lane, and T.-M. Yan, Phys. Rev. D 21, 203 (1980).

[81] J. S. Ball and T. W. Chiu, Phys. Rev. D 22, 2542 (1980); 22, 2550 (1980).

[82] M. Cheng, N. H. Christ, S. Datta, J. van der Heide, C. Jung, F. Karsch et al., Phys. Rev. D 77, 014511 (2008).

[83] A. Bazavov, T. Bhattacharya, C. Detar, H.-T. Ding, S. Gottlieb, R. Gupta et al., Phys. Rev. D 90, 094503 (2014).

[84] S. Borsanyi, Z. Fodor, C. Hoelbling, S. D. Katz, S. Krieg, and K. K. Szabo, Phys. Lett. B 730, 99 (2014). 